\title{
A novel nanofabrication technique using focused ion beam (FIB), metal organic chemical vapour deposition (MOCVD)
}

\author{
Wasim Haskiya \\ Irish Robotics Academy, Ireland \\ Email address: \\ Irish.robotics.academy@hotmail.com
}

\section{To cite this article:}

Wasim Haskiya. A Novel Nanofabrication Technique Using Focused Ion Beam (FIB), Metal Organic Chemical Vapour Deposition (MOCVD). American Journal of Nanoscience and Nanotechnology. Vol. 1, No. 2, 2013, pp. 46-51. doi: 10.11648/j.nano.20130102.11

\begin{abstract}
The aim of this paper is to present a novel nano-manufacturing technique for the fabrication of nano-scale systems, such as mechanical machines and printed circuits etc. The proposed technique utilizes a guided focused ion beam (FIB) through a pattern to a substrate where it decomposes a metal organic gas to generate a reduced outline copy of the mask, yielding the required design system. The novelty of this technique is in the ability to fabricate nano-scale systems layer-by-layer rather than atom-by-atom through the adjustment of the vertical position of the fabricated part.To demonstrate the proposed technique, a simulation model was designed and tested. The simulation results have shown that a reduction in the perimeter of the fabricated part can be achieved easily by adjusting its vertical position with respect to the beam focused point by a 100 to 400 times. Further investigation revealed that the growth rate is a function of the precursor flux. For example, using a titanium precursor flux below the $2 \times 10^{10}$ molecules $/ \mathrm{cm}^{2} . s$ then sputtering is more predominant, and above that value, a net deposition will occur at a growth rate of $5.3 \times 10^{-05} \mathrm{~cm} / \mathrm{s}$.
\end{abstract}

Keywords: Nanomanufactiring, FIB, Metal Organic Gas, Outline Features, Precursor Flux

\section{Introduction}

At present, nanoscale manufacturing is categorized to either top-down or, bottom-up techniques[1]. The topdown manufacturing process of nano structures involves either additive or subtractive of material, such as wet/dry etching, or the deposition of a thin film on a silicon based wafer. This technology is known as Nanomachining (NEMS), which is an extension to micromachining (MEMS) on a typical top-down approach (from large to small). On the other hand, bottom-up manufacturing processes go from small to large (from atom-up). Therefore these methods are often too slow to be practical. To attain a more acceptable building speed, self assembly, replication chemistry, and the use of some what larger building blocks (e.g. cluster of atoms) are often essentials. These techniques are referred to as Nanochemistry[2]. Important tools in nanochemistry are the Atomic Force Microscope (AFM) and the Scanning Tunneling Microscope (STM). These microscopes employ a probe[3] having sharp tip to manipulate atoms one at a time to build a structure. For this reason, Nanochemistry is also referred to as Molecular manufacturing. Whether NEMS or Molecular manufacturing these technologies are not compatible with high volume production, besides, they are time consuming, which results in high production cost.

On the other hand, the objective of this research is to develop a manufacturing technology for the nanometer features/structures utilizing a focused ion beam (FIB), and Metal Organic Chemical Vapour Deposition processes.

\section{Description}

Figure (1) shows a schematic illustration of the proposed nanofabrication technique. A focused-ion-beam (FIB) is bombard in a vacuum to a specific point called; i-beam focused point (BFP). Using existing lithography techniques, a patterned mask is made with the required design outline's feature, and positioned on a stage right below the scanned i-beam source.

A second $\mathrm{X}-\mathrm{Y}-\mathrm{Z}$ positioning stage is placed between the mask, and the BFP, in which an oxidized silicon wafer is placed on it. All four elements; i-beam source, mask stage, $\mathrm{SiO}_{2}$ positioning stage and the $\mathrm{BFP}$ are aligned to one 
perpendicular axis. In the meantime, a metal organic precursor gas is spread uniformly on the surface of the substrate through a nozzle where they are adsorbed. Then, a FIB is scanned through the mask outline to decompose the adsorbed precursor gas, leaving a metal film on the substrate surface, which is a reduced copy of the mask feature's outline. The volatile product from the above reaction is taken care by the vacuum pump.

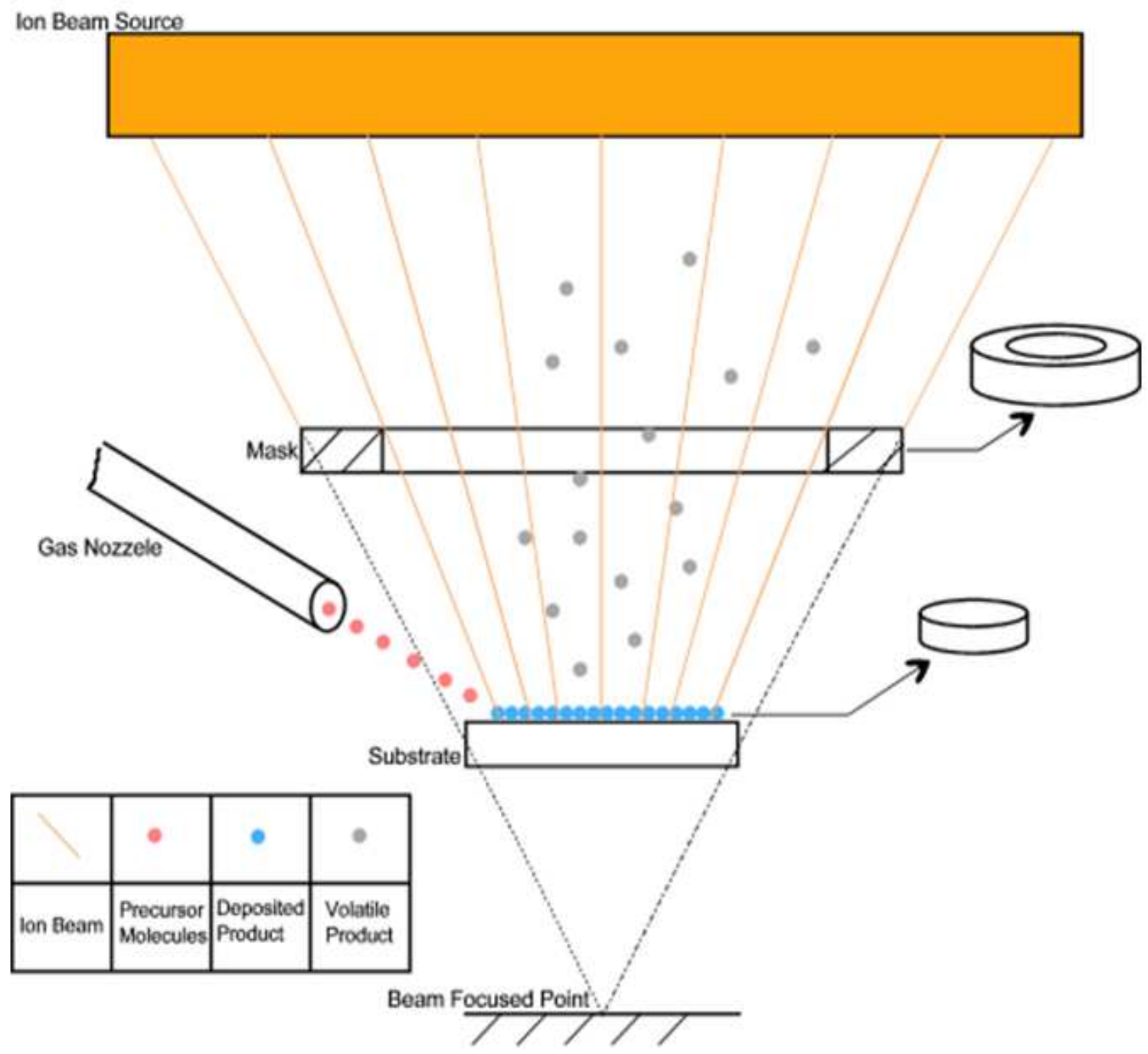

Figure 1. Schematic illustration of the proposed nano-fabrication using FIB

By adjusting the vertical position of $\mathrm{SiO}_{2}$ wafer with respect to the $\mathrm{BFP}$, one can reduce the size of the new fabricated part or features. Eventually, this technique can generate features that are hundreds of times smaller than the original design of the patterned mask.

\section{Modeling}

To demonstrate the proposed technique; a mathematical expression governing the relationship between the initial size of the mask to that of a new generated feature/structure is formulated.

$$
r_{n}=\frac{h}{H_{m}} r_{m}=\frac{h}{H_{b}} r_{b}
$$

Eq. 1 is a geometrical relationship that gives the maximum size of the fabricated part. But in reality; the new part size is a function to ion beam parameters and ion source accelerator. Thus according to reference[4] the maximum ion beam current that can be accelerated is approximately:

$$
\begin{aligned}
& I=\frac{\left(\frac{4 \varepsilon_{0}}{9}\right) A_{b} \sqrt{\frac{e}{m}} V_{t}^{\frac{3}{2}}}{l_{g}^{2}} \\
& I=\frac{\left(\frac{4 \varepsilon_{0}}{9}\right) \pi r_{b}^{2} \sqrt{\frac{e}{m}} V_{t}^{\frac{3}{2}}}{l_{g}^{2}} .
\end{aligned}
$$

Where $\varepsilon_{0}$ is the permittivity of space, $A_{b}$ and $r_{b}$ are the beam area and radius, $e / m$ is the charge to mass ratio of the accelerated ions, $V_{t}$ is the total voltage, and $l_{g}$ is the gap 
between the screen and accelerator grid. Solving for beam radius will yield:

$$
r_{b}=\sqrt{\frac{I}{\frac{\pi\left(\frac{4 \varepsilon_{0}}{9}\right) \sqrt{\frac{e}{m}} V_{t}^{\frac{3}{2}}}{l_{g}^{2}}}}
$$

Now substitute eq. 3 , in eq. 1 will give the size of the newly fabricated part as a function to ion beam parameters:

$$
r_{n}=\frac{h}{H_{b}} \sqrt{\frac{\pi\left(\frac{4 \varepsilon_{0}}{9}\right) \sqrt{\frac{e}{m}} V_{t}^{\frac{3}{2}}}{l_{g}^{2}}}
$$

So eq. 4 gives the size of the fabricated part in the horizontal plane.

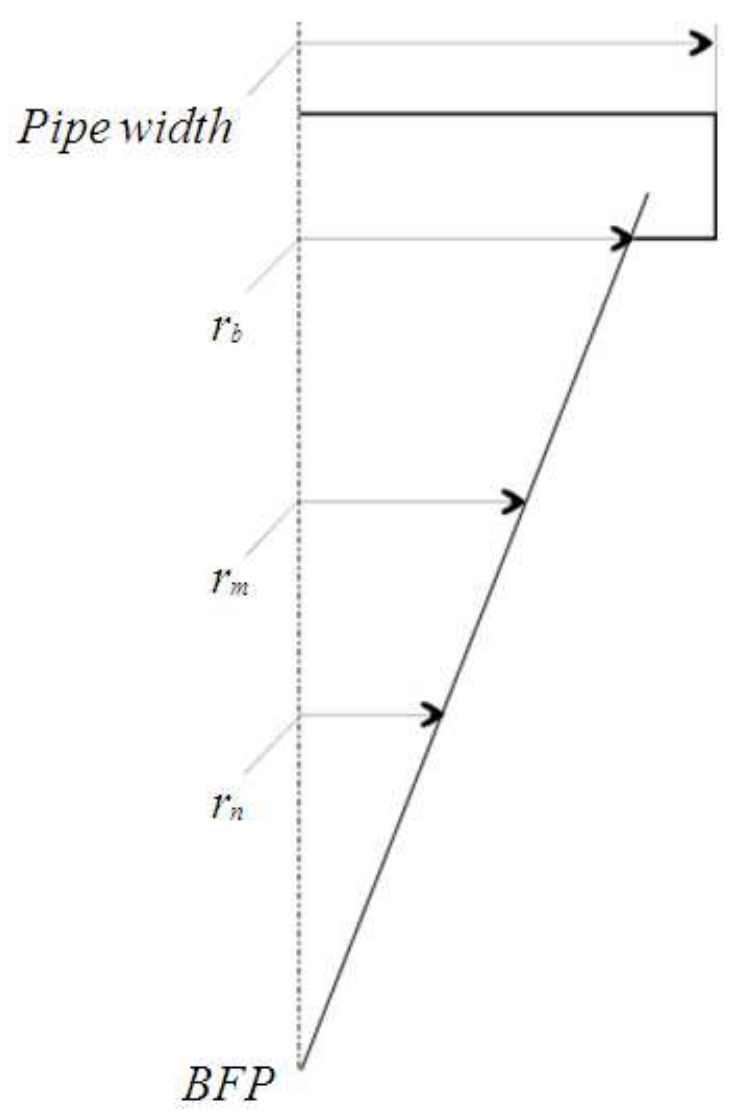

Figure 2. Geometrical relation of system parameters

Figure (3) illustrate a collision cascade model for the fabrication mechanism, and it can be summarized in three main steps: deposition of precursor gas molecules on substrate; ions bombard through the mask pattern to the hit adsorbed molecules and decompose them; and finally sputtering. The size of the newly fabricated part is characterized by the deposition yield (YN), which is the net number of deposited atoms/molecules per incident ion. The decomposition yield ( $\mathrm{YD}=\mathrm{N} \sigma$ ), expressed as the product of the surface coverage by the adsorbed precursor $\mathrm{N}$ (molecules $/ \mathrm{cm} 2)$ and the dissociation cross section $\sigma(\mathrm{cm} 2)$. The sputtering yield (YS), which is the number of atoms sputtered per incident ion.

To express this in terms of balance between decomposition and sputtering, the net deposition yield (YN)[5] is the difference between the decomposition yield and the sputtering yield (eq. 5).

$$
Y_{N}=Y_{D}-Y_{S}
$$

The actual deposition process is described as a change of density of the adsorbed precursor gas to the substrate[6-8]. The key elements involved during deposition are: $(a)$ adsorption of precursor gas, governed by the gas flux $F$ and the sticking coefficient $g ;(b)$ molecules decomposition governed by the product $N \sigma$ due to the current density $J$ of the ion beam; (c) spontaneous sputtering of atoms after a residence time $\tau$. So, the change of density to the adsorbed precursor can be defined as a rate of adsorption of precursor on substrate minus decomposition rate of precursor molecules due to ion beam minus the sputtering molecules/atoms due to the ion beam, and this is described in eq. 6.

$$
\frac{d N}{d t}=g F\left(1-\frac{N}{N_{0}}\right)-\sigma N J-\frac{N}{\tau}
$$

It has been proved by references[9-11] that a steady state condition of $N$ can be reached when $d N / d t=0$. Then solving eq. 6 for the steady state condition would yield eq. 7 .

$$
N=\frac{g F}{\frac{g F}{N_{0}}+\sigma J+\frac{1}{\tau}}
$$

Where N0 is the available adsorption site density in a monolayer.

According to figure (3), and further to eq. 5, the deposition yield YN can be expressed as deposited volume of film per unit charge[12, 13]. Where the film volume is the cross section A time depth/deposition height sz:

$$
Y_{N}=\frac{A s_{z}}{I t}
$$

Where I and t: the ion current, and the total deposition time.

The sputtering yield YS of eq. 5, can be calculated from the sputtered rate $\mathrm{Yr}$ that is an experimental value measured for different materials[14]. Then the relationship is:

$$
Y_{S}=96.4 \frac{\rho Y_{r}}{m}
$$

Where $m$ is the mass in (amu), and $\rho$ is the density in $\left(\mathrm{g} / \mathrm{cm}^{3}\right)$. 


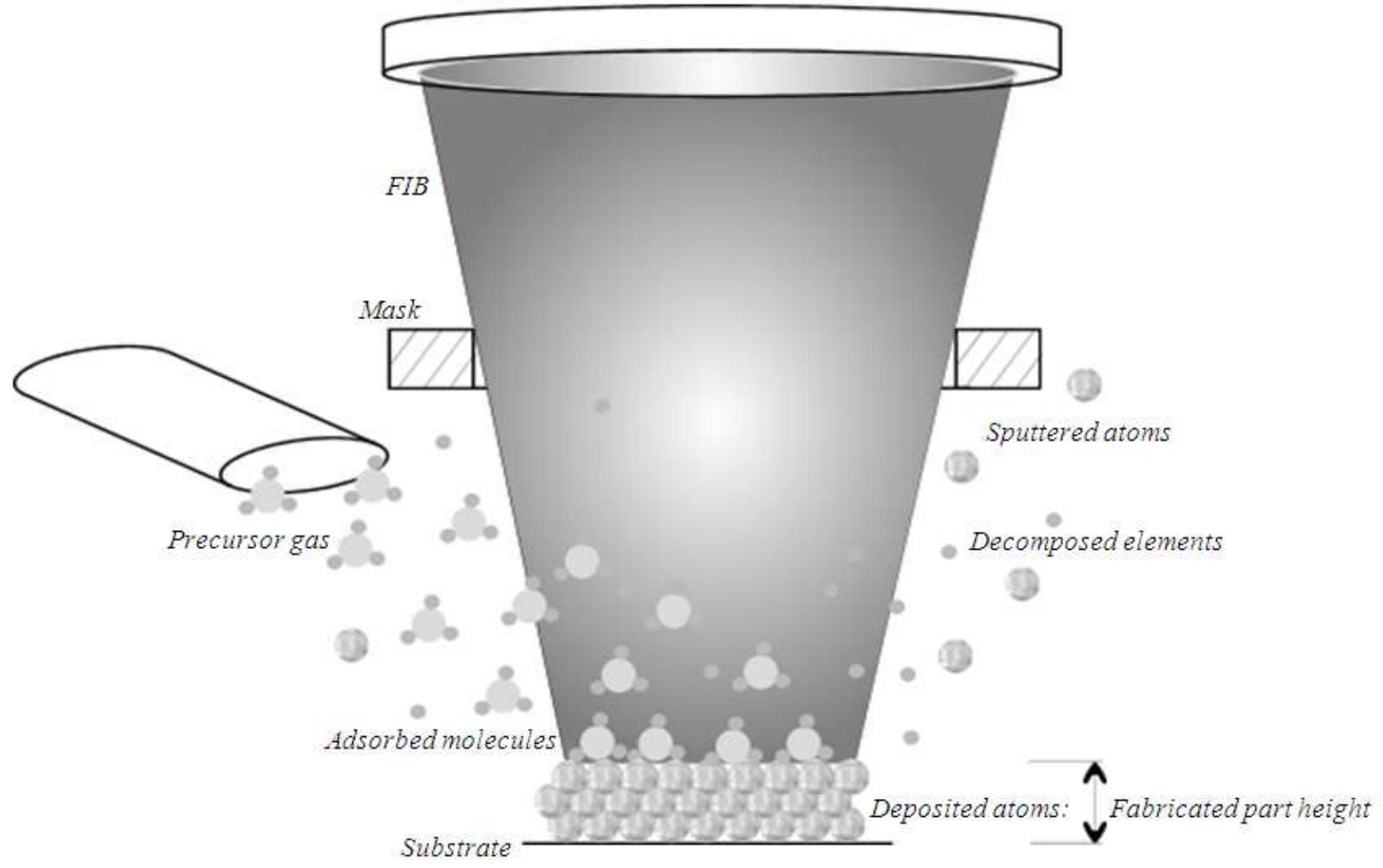

Figure 3. The collision cascade model yields the thickness of a newly fabricated part

Now, to find the growth rate and or new product thickness, substitute eq. 6,8 , and 9 , in eq. 5 will yield:

$$
\begin{aligned}
& \frac{A s_{z}}{I t}=\left(\frac{g F}{\frac{g F}{N_{0}}+\sigma J+\frac{1}{\tau}}\right) \sigma-96.4 \frac{\rho Y_{r}}{m}, \text { then } \\
& \frac{s_{z}}{t}=\frac{I}{A}\left[\left(\frac{g F}{\frac{g F}{N_{0}}+\sigma J+\frac{1}{\tau}}\right) \sigma-96.4 \frac{\rho Y_{r}}{m}\right], \text { or } \\
& s_{z}=\frac{I t}{A}\left[\left(\frac{g F}{\frac{g F}{N_{0}}+\sigma J+\frac{1}{\tau}}\right) \sigma-96.4 \frac{\rho Y_{r}}{m}\right]
\end{aligned}
$$

As shown in eq. 10, the growth rate and or product height is a function of the variable parameters; beam current/density, precursor flux, dissociation cross section and the available adsorption site density in a monolayer. Eq. 10 , is demonstrated in the following section.

\section{Testing and Simulation}

To demonstrate the proposed nanofabrication technique, typical commercial ion source parameters along with Titanium precursor are used in the proposed model.

The simulation model assumes different design approaches:

- The size of the fabricated part is known, then find optimal parameters;

- A fixed energy/current, but part positioning is variable;

- A fixed part positioning, but variable energy/current

Typical values for an LMIS (e.g. FEI200 series)[15]; distance between source and BFP is $0.5 \mathrm{~m}$, source diameter $0.15 \mathrm{~m}$, acceleration gap $0.001 \mathrm{~m}$, current density $0.1 \mu \mathrm{A} / \mathrm{cm}^{2}$ to $10 \mathrm{~A} / \mathrm{cm}^{2}$, energy $25 \mathrm{KeV}$, and the Gallium dissociation cross section is $6.5 \mathrm{~nm}^{2}$.

The initial simulation results have proved that the closer the new part position to the BFP the greater in size reduction can be achieved.

Also, at a current of $5 \mathrm{nA}$ and higher, there is a decrease in the slope along with an increase in the part size in proportion to the fed current.

From the geometrical point of view; positioning the mask greater than the $10 \mathrm{~mm}$ away from the BFP, will result 
in a greater reduction in the part size, compared to the mask.
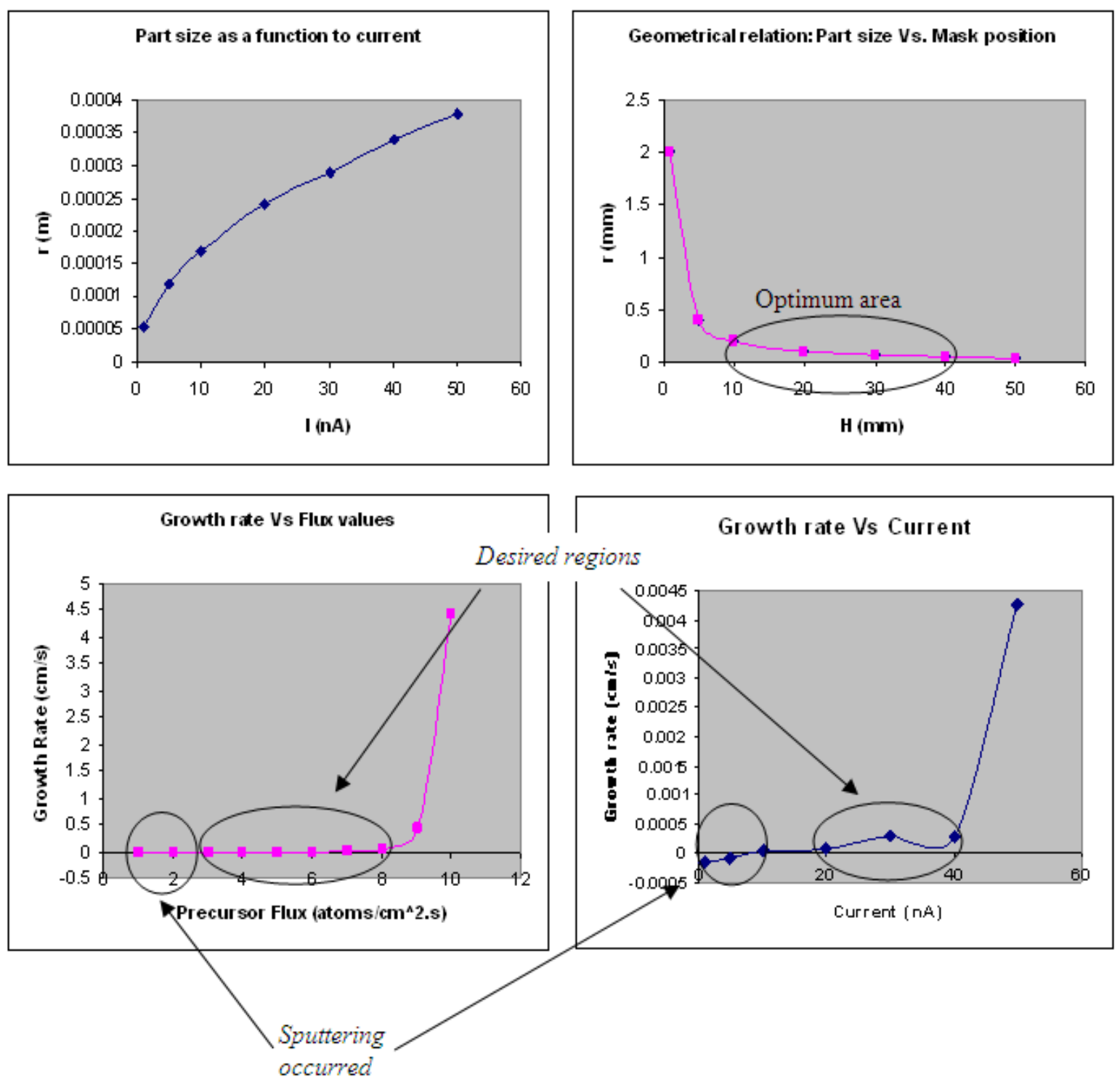

Figure 4. Summary of simulation results

Furthermore, the growth rate is a function of the precursor flux. For example, when using a titanium precursor with flux lower than $2 \times 10^{10}$ molecules $/ \mathrm{cm}^{2} . \mathrm{s}$, then sputtering is more predominant, and above that value a net deposition will occur at a growth rate of $5.38 \times 10^{-05} \mathrm{~cm} / \mathrm{s}$. Also, for optimum process, the fed current should be between 20 to $40 n A$.

\section{Conclusion}

A novel technique for the manufacturing at the nanoscale has been presented and demonstrated. The theoretical analysis and simulation results have proved its capabilities in achieving a reduction in part/feature size by 100 to 400 times compared to original mask size. For optimum results, the current should be between 10 to $40 \mathrm{nA}$, and the flux between $2 \times 1010$ to 1011 atoms $/ \mathrm{cm} 2 . \mathrm{s}$, then a net deposition occurs at a growth rate $5.38 \times 10-5$ to $3 \times 10-4 \mathrm{~cm} / \mathrm{s}$. Below the 2x1010 atoms $/ \mathrm{cm} 2 . s$ sputtering is dominant.

\section{References}

[1] Marc J. Madou, Fundamentals of microfabrication - the science of miniaturization, CRC Press, Second eddition

[2] Edward L. Wolf, Nanophysics and nanotechnology: An introduction to modern concepts in nanoscience, Physics textbook, WILEY-VCH

[3] Michael Rieth, Nano-engineering in science and technology: an introduction to the world of nano design, World scientific, Vol. 6 
[4] Stanley Humphries, Jr., Charged particle beams, Originally published in 1990 by John Wiley and Sons (QC786.H86 1990, ISBN 0-471-60014-8)

[5] John Melngailis, Focused ion beam fabrication of microelectronic structures, Final report - U.S. Army Research office Contract DAALO3-87-K-0126, Research Laboratory of Electronics Massachusetts Institute of Technology

[6] Fabio Cicoira, Electron beam induced deposition of rhodium Nanostructures, École Polytechnique Fédérale De Lausanne, Thèse No 2528 (2002)

[7] Yongqi Fu, Ngoi Kok, Ann Bryan, Ong Nan Shing, Characterization of focused ion beam induced deposition process and parameters calibration, Elsevier Sensors and actuators A 88 (2001) 58-66

[8] Joon Hyun Kim, Youn-Jea Kim, Influence of stage control parameters on pattering in the focused ion beam deposition process, Journal of the Korean Physical Society, Vol. 53, (Nov. 2008), 2596-2602

[9] B. W. Kemshall, L. A. Giannuzzi, B. I. Prenitzer, F. A. Stevie, S. X. Da, Comparative evaluation of protective coatings and focused ion beam chemical vapor deposition process, Journal of the American Vacuum Society, B20(1), Jan/Feb 2002, 286-290
[10] Jack Zhou, Guoliang Yang, Focused ion beam based nanohole modeling, simulation, fabrication, and application, Journal of manufacturing science and engineering, Vol. 132, Feb 2010, 011005-1 to 011005-8 (Transactions of the ASME)

[11] Jerome J. Cuomo, Stephen M. Rossnagel, Harold R. Kaufman, Handbook of ion beam processing technology principles, deposition, film modification and synthesis, Noyes publications

[12] Ivo Utke, Pattrick Hoffmann, John Melngailis, Gas-assisted focused electron beam and ion beam processing and fabrication, J. Vac. Sci. Technol. B, Vol. 26, No. 4, Jul/Aug $2008,1197-1276$

[13] Francis L. Ross III, Nano-cellural microstrtucture evolution in ion induced chemical vapour deposition (II-CVD) of copper, Submitted to the department of material science and engineering in fulfillment of the requirement of the degree Doctor of Philosophy at the, Massachusetts Institute of Technology, Sep. 2003

[14] Jon Orloff, Handbook of charged particle optics, second edition, CRC Press

[15] Edited by: Bernhard Wolf, Handbook of Ion sources, CRC 